DOI 10.35381/cm.v5i9.292

\title{
Las Competencias Digitales y la Integración Pedagógica de las TIC en Docentes
}

\section{Digital Competences and Pedagogical Integration of ICT in Teachers}

\author{
Renzo Cuarez Cordero \\ renzocuarez@hotmail.com \\ Universidad Nacional Mayor de San Marcos, Lima \\ Perú \\ https://orcid.org/0000-0003-4173-2876
}

Recibido: 05 de mayo de 2019

Aprobado: 10 de junio de 2019

\section{RESUMEN}

La investigación tuvo por objetivo establecer la relación entre las competencias digitales y la integración pedagógica de las TIC en docentes participantes del proyecto 2235483 de la ciudad de Lima Metropolitana en el año 2019. Metodológicamente fue de tipo correlacional, la muestra poblacional estuvo conformada por 45 docentes, se les procesó un instrumento de medición para la Integración pedagógica de las TIC, de 20 ítems y en escalamiento de Likert de cinco alternativas de repuestas, el cual fue validado por el juicio de tres expertos y obteniendo un coeficiente de confiabilidad de Cronbach de 0.902 catalogándose como altamente confiable para su aplicación. Al existir significancia bilateral de 0,793 es mucho mayor que el parámetro de confianza 0.05 lo que procede a rechazarse la hipótesis afirmativa $(\mathrm{H} 1)$ y se acepta la nula $(\mathrm{H} 0)$.

Descriptores: tecnología de la información; tecnología educacional; Informática educativa; enseñanza asistida por ordenador.

\section{ABSTRACT}

The objective of the research was to establish the relationship between digital skills and the pedagogical integration of ICT in teachers participating in the project 2235483 of the city of Metropolitan Lima in the year 2019. Methodologically it was correlational, the population sample consisted of 45 teachers, they were processed a measuring 
instrument for the pedagogical Integration of ICT, of 20 items and in Likert scaling of five alternative answers, which was validated by the judgment of three experts and obtaining a Cronbach reliability coefficient of 0.902 categorized as highly reliable for its application. Since there is a bilateral significance of 0.793 , the affirmative hypothesis $(\mathrm{H} 1)$ is rejected much more than the 0.05 confidence parameter and the null $(\mathrm{HO})$ is accepted.

Descriptors: information technology; educational technology; computer uses in education, computer assisted instruction.

\section{INTRODUCCIÓN}

La sociedad actual se caracteriza, básicamente por la demanda de grandes cantidades de información y conocimiento, de ahí que viene las frases sociedad de la información y del conocimiento, es decir, se refiere a la interconectividad e interacción, gran producción y elaboración de información que proviene de distintas y variadas bases, bisoñas y especializadas. El acceso a esta información con el paso del tiempo se ha vuelto cada vez más sencillo, todo esto gracias a que una gran cantidad de dicha información se encuentra digitalizada y las poblaciones que, en su mayoría son jóvenes, (esto no quiere decir que no los adultos) utilizan el internet para indagar en los registradores para enterarse de temas que son de su interés. Al respecto Palomino, citado por Zevallos (2018) menciona que para las generaciones que nacen en la era digital, "desenvolverse en los entornos virtuales suele ser sencillo, pero pocas veces es usado para fines de aprendizaje, para los adultos de generaciones anteriores a la era digital, estos entornos pueden presentar más retos que beneficios debido a su poca alfabetización digital" (p. 48).

Por lo sostenido en líneas arriba, los responsables y expertos de la educación se encuentran en la gran necesidad, por no decirlo en la obligación de desarrollar las competencias digitales, ya que son ellos quienes van orientar a los estudiantes para que puedan desarrollar su ciudadanía digital de manera responsable y las demás habilidades relacionadas a las TIC. 
La integración de las TIC es un largo proceso, en nuestro país todavía existe una gran carencia en su implementación, ya que gran parte de los sectores educativos no cuentan los medios básicos (computadoras, internet, etc.), en los sectores rurales, por ejemplo, se requiere con urgencia "de la integración de las TIC" para que de esta manera la educación no sea tan inequitativa.

Además es importante mencionar que, para la integración pedagógica de las TIC, es necesario que se cuente con docentes altamente capacitados en los temas, para ello, se necesita cambiar el enfoque formativo de los mismos, si bien, en el proceso formativo de la profesión, se llevan cursos relacionados a las TIC, pero no son desarrollados de manera adecuada, ya que todavía se tiene la idea errónea de que las TIC solamente sirven para compartir y transmitir información, entonces, se debe cambiar esa concepción.

Los estudiantes de hoy, han nacido en la era de la tecnología, por ende tienen nuevas formas de aprender, buscan otras maneras de aprender, esto es mediante el avance tecnológico, y los docentes, deben estar preparados para afrontar este nuevo reto de la educación, esto se logrará mediante el desarrollo de sus competencias digitales.

Existe un gran problema al que los docentes se tienen que enfrentar hoy en día y tiene que ver con la metodología que emplean en el aula. "La clase frontal, donde el docente es el centro de atención, va en franca decadencia, siendo poco a poco menos utilizada. Es así que en la sala de clases se busca la construcción del conocimiento del mundo en que vivimos", Britio, Jara Salazar \& Larenas (2009). Cada docente tiene sus propios regalas, y sus propios modelos mentales, los cuales permiten que dé sentidos a sus experiencias y a las actividades que realiza.

Por otro lado, el agigantado avance de las tecnologías, ofrece a los docentes más y mejores medios o alternativas para realizar múltiples actividades, esto incluye las actividades en las aulas, en el caso de los docentes. Además, los estudiantes de estos tiempos ya están circunscritos en la era tecnológica, es decir, han nacido en el creciente aumento de la tecnología, y por ende, se tiene la certeza de que asimilan de 
una mejor manera los contenidos y conocimientos que son compartidos a través de los medios tecnológicos.

De ahí que Britio, Jara Salazar \& Larenas (2009), sostienen "el profesor de hoy se enfrenta a la encrucijada de superar el tedio que se advierte en los estudiantes ante una clase expositiva o bien, a través de medios tecnológicos informáticos multimediales interactivos, hacerla más interactiva" (p. 7). Por todo lo anterior, es necesario y urgente que los docentes en formación y en actividad, empiecen a desarrollar sus competencias digitales para enfrentar los retos de la nueva era digital y de la educación requerida para el siglo XXI.

Para llegar a entender el proceso de integración pedagógica de las TIC es importante plantear o establecer la base conceptual, para ello se recurrirá a diversos autores como Sánchez citado por Parra \& Pincheira (2019) quien define a la integración pedagógica de las TIC como el:

Proceso de hacerlas enteramente parte del currículo, como parte de un todo, permeándolas con los principios educativos y la didáctica que conforman el engranaje del aprender. Ello fundamentalmente implica un uso armónico y funcional para un propósito del aprender específico en un dominio (p. 3).

En esta misma línea, Grabe citado por el, mismo autor, manifiesta que la integración pedagógica de las TIC se da "cuando las TIC ensamblan confortablemente con los planes instruccionales del profesor y representa una extensión y no una alternativa o una adición a ellas", Parra \& Pincheira (2019). Actualmente, por el masivo y vertiginoso avance de la tecnología no es necesario, sino fundamental la integración de las TIC en la educación, ya que las nuevas generaciones crecen en un contexto muy distinto a las generaciones anteriores, los intereses de estas generaciones son los medios tecnológicos, de ahí que para alcanzar los objetivos de la educación del siglo XXI se requiere de medios innovadores y qué mejor que las TIC para poder llegar a los estudiantes, en este sentido, Parra \& Pincheira (2019), mencionan al respecto:

El desarrollo de la Sociedad de la Información, caracterizada por el uso masivo y creciente de las TIC en cada uno de los aspectos del ser 
humano y por una fuerte tendencia a la globalización económica y cultural, exige que los habitantes de esta sociedad, desarrollen nuevas competencias para poder afrontar con éxito los cambios que impone el vertiginoso avance de la tecnología, para así ser incluido como ciudadano de la sociedad del conocimiento. (p. 3)

Los cambios de los que hablan los autores han impactado enormemente en el campo educativo. Es por ello que esta nueva era cultural reclama que se planteen nuevas maneras de ver y entender el mundo, brindando diferentes medios para entablar las comunicaciones a nivel social como interpersonal, arrasando con las barreras del tiempo y de la distancia o espacio, enlazando de manera instantánea y universal a todas las personas del mundo, además de facilitar a la vez, las actividades o labores tradicionales, mediante renovadas herramientas tecnológicas, que optimizan la producción y que, son acogidas rápidamente por esta generación de estudiantes que nacen con la tecnología.

En este sentido Marqués citado por Parra \& Pincheira (2019), cuando habla de las competencias básicas en TIC que son importantes para los docentes, plantea la articulación entre la tecnología y el proceso de formación, al respecto sostienen:

Las TIC se han convertido en un eje transversal de toda acción formativa donde casi siempre tendrán una triple función: como instrumento facilitador los procesos de aprendizaje (fuente de información, canal de comunicación entre formadores y estudiantes, recurso didáctico...), como herramienta para el proceso de la información y como contenido implícito de aprendizaje (los estudiantes al utilizar las TIC aprenden sobre ellas, aumentando sus competencias digitales). (p. 3).

Al considerar este planteamiento, el autor fundamenta la integración de la TIC en el proceso educativo mediante tres pilares preeminentes, los cuales se detallan a continuación:

Primeramente, se debe tener en cuenta y "reconocer la disponibilidad y facilidad de acceso que ofrece actualmente el internet, brindándonos grandes cantidades de información y conocimientos" (Parra \& Pincheira, 2019). 
En segundo lugar, "la gran posibilidad que ofrecen las TIC para modificar, potenciar y actualizar de manera constante y relativamente a bajo costo los ambientes de aprendizaje en que los educandos se encuentran insertos" (Parra \& Pincheira, 2019). En último lugar:

El tercer pilar hace referencia a la necesidad de desarrollar la competencia TIC para la docencia, de manera tal que el educador sea capaz de responder a las nuevas demandas de conocimiento que en los distintos campos del quehacer humano, ha generado el uso y abuso de las TIC en la sociedad postmoderna" (Parra \& Pincheira, 2019, p. 4).

Para cerrar este punto se puede afirmar que, la integración de las TIC en la educación es fundamental si se quiere cambiar verdaderamente el modelo educativo que todavía impera en nuestros días. La generación venidera lo exige, por ende, el Estado debe implementar los medios necesarios para poder preparar el ambiente que se requiere. Además de la implementación de los medios, es importante empezar ya, la capacitación de los docentes en ejercicio y de los docentes en formación, esto no sola a un parte, sino que se debe iniciar, a nivel nacional la implementación y capacitación a los docentes, en temas relacionados al uso de las TIC en la educación.

\section{MÉTODO}

La investigación de tipo descriptivo correlacional por cuanto el objetivo general: establecer la relación entre las competencias digitales y la integración pedagógica de las TIC en docentes participantes del proyecto 2235483 de la ciudad de Lima Metropolitana en el año 2019, para lo cual se prosiguió con lo establecido metodológicamente por Hernández, Fernández \& Baptista (2014), quienes explican que la correlación permite establecer la relación o no entre dos variables. La muestra poblacional estuvo conformada por 45 docentes participantes del proyecto 2235483 de la ciudad de Lima Metropolitana en el año 2019, a quienes se les procesó un instrumento de medición para la Integración pedagógica de las TIC, basado en 20 ítems 
y en escalamiento de Likert de cinco alternativas de repuestas, el cual fue validado por el juicio de tres expertos y obteniendo un coeficiente de confiabilidad de Cronbach de 0.902 catalogándose como altamente confiable para su aplicación. Estadísticamente se aplicó correlación de Spearman, el cual permitió establecer la relación o no de las variables, mediante la aplicación del programa SPSS versión 25, y teniéndose como referencia el baremo de correlación propuesto por Hernández, Fernández y Baptista (2014):

Interpretación: el coeficiente $r$ de Pearson puede variar de $-1.00 \mathrm{a}+1.00$, donde:

-1.00 =correlación negativa perfecta. ("A mayor $X$, menor $Y$ ", de manera proporcional. Es decir, cada vez que $X$ aumenta una unidad, $Y$ disminuye siempre una cantidad constante). Esto también se aplica "a menor $\mathrm{X}$, mayor $\mathrm{Y}$ ".

$-0.90=$ Correlación negativa muy fuerte .

$-0.75=$ Correlación negativa considerable.

$-0.50=$ Correlación negativa media .

-0.25 = Correlación negativa débil.

$-0.10=$ Correlación negativa muy débil.

$0.00=$ No existe correlación alguna entre las variables.

$+0.10=$ Correlación positiva muy débil.

+0.25 = Correlación positiva débil.

$+0.50=$ Correlación positiva media.

+0.75 = Correlación positiva considerable .

$+0.90=$ Correlación positiva muy fuerte.

+1.00 =Correlación positiva perfecta ("A mayor $X$, mayor $Y$ " o "a menor $X$, menor $Y$ ", de manera proporcional. Cada vez que $X$ aumenta, $Y$ aumenta siempre una cantidad constante). 


\section{RESULTADOS}

\section{Cuadro 1}

Correlación entre competencias digitales y la integración pedagógica de las TIC

\begin{tabular}{|c|c|c|c|}
\hline & & $\begin{array}{c}\text { El Competencias } \\
\text { digitales }(X)\end{array}$ & $\begin{array}{c}\text { Integración } \\
\text { pedagógica }(\mathrm{Y})\end{array}$ \\
\hline \multirow{3}{*}{$\begin{array}{c}\text { El Competencias } \\
\text { digitales }(X)\end{array}$} & $\begin{array}{l}\text { Correlación } \\
\text { de Pearson }\end{array}$ & 1 & 0,040 \\
\hline & $\begin{array}{l}\text { Sig. } \\
\text { (bilateral) }\end{array}$ & & 0,793 \\
\hline & $\mathrm{N}$ & 45 & 45 \\
\hline \multirow{3}{*}{$\begin{array}{c}\text { Integración } \\
\text { pedagógica }(\mathrm{Y})\end{array}$} & $\begin{array}{l}\text { Correlación } \\
\text { de Pearson }\end{array}$ & 0,040 & 1 \\
\hline & $\begin{array}{l}\text { Sig. } \\
\text { (bilateral) }\end{array}$ & 0,793 & \\
\hline & $\mathrm{N}$ & 45 & $45^{\prime \prime}$ \\
\hline
\end{tabular}

Al generarse una correlación de 0,040 se procede a catalogar como una correlación positiva débil en función del baremo planteado por Hernández, Fernández y Baptista (2014), lo cual implica que existe relación entre las variables, sin embargo, al ser positiva débil, se evidencia que pudieran existir factores que afectan el fortalecimiento relacional de las variables.

Así mismo, al existir significancia bilateral de 0,793 es mucho mayor que el parámetro de confianza 0.05 lo que procede a rechazarse la hipótesis afirmativa $(\mathrm{H} 1)$ y se acepta la nula $(\mathrm{H} 0)$, declaradas del siguiente modo:

$\mathrm{H} \mathrm{O}=$ No existe relación lineal y directa entre las competencias digitales y la integración pedagógica de las TIC en docentes participantes del proyecto 2235483 de la ciudad de Lima Metropolitana en el año 2019.

H1= Existe relación lineal y directa entre las competencias digitales y la integración pedagógica de las TIC en docentes participantes del proyecto 2235483 de la ciudad de Lima Metropolitana en el año 2019. 


\section{DISCUSIÓN}

Al rechazarse la hipótesis positiva y al existir correlación positiva débil, implica que existen factores intervinientes en el proceso de relación de las variables, lo cual se puede tomar como la necesidad de fortalecer aspectos que contribuyan a la generación de una relación lineal y significativa de las variables, lo cual no se deba al azar o a procesos no derivados de la aplicación sistemática de medidas para el fortalecimiento de las competencias abordadas en la investigación, siendo pertinente tener en cuenta lo planteado por Fernández Cruz, Fernández Díaz \& Rodríguez Mantilla (2018), quienes indican que:

Los aspectos que afectan en mayor grado al proceso de implementación e integración de las tecnologías en un centro educativo son otros: formación en competencias digitales, coordinación tecno-pedagógica, el uso pedagógico diseñado desde el currículo escolar y liderazgo efectivo que genere un clima de aceptación de las tecnologías como elemento de innovación educativa (p. 412).

Son diversos factores que se encuentran presentes en la conformación de las competencias digitales y la integración pedagógica de las TIC, siendo pertinente promover la formación permanente en los docentes como una estrategia que permita progresivamente empoderar las variables de investigación en función de fortalecer el uso efectivo de las herramientas digitales y TIC con fines educativos, lo cual podría fortalecer la correlación en próximas investigaciones.

Por otro lado, Gómez (2017), enfatiza la importancia de que los docentes además adopten la planificación curricular desde la investigación, lo cual permitirá establecer mayores posibilidades de promover la aceptación efectiva de las TIC en la educación con pertinencia a generar la focalización de asumir desde la investigación la consolidación de un enfoque pedagógico que puede encontrar resistencia en los docentes para su debida implementación, por cuanto el docente investigador concibe en el horizonte educativo como un reto para crecer integralmente, lo cual le permite aprender para afrontar retos educativos y pedagógicos. 
Así es necesario generar un cambio transformacional en la gerencia educativa con la finalidad de promover el empoderamiento de las nuevas tendencias pedagógicas basadas en la tecnología, Zurita (2018), comenta la necesidad de involucrar a la comunidad educativa en su totalidad con la finalidad de generar la sinergia pertinente que permita trabajar cooperativamente en la consolidación de afrontar con eficacia los modelos educativos con tendencia hacia la educación 2030 , así el protagonismo no solo recaerá en el docente, sino, en el equipo que conforma la institución educativa.

\section{CONCLUSIONES}

Es necesario realizar nuevas mediciones estadística, con la finalidad de conocer los factores que podrían estar afectando en la generación de la aceptación de la hipótesis nula, por cuanto existen diversos elementos como la falta de formación permanente en el docente, así como la aplicación de la investigación como eje neurálgico para el fomento educativo que no se estén desarrollando efectivamente.

Siendo pertinente además que otros investigadores no solo se basen en el hecho lineal de medir las competencias digitales y TIC, debido que entre ambas variables se encuentran implícitas diversos factores que podrían afectar el buen o mal funcionamiento de ambos, considerando necesaria extrapolar a poblaciones similares la medición estadística generada con la finalidad de comparar la efectividad de la relación de ambas variables.

Proseguir con la generación de investigaciones para conocer el comportamiento individual y relacional de las variables, podrá fortalecer las conclusiones en función de construir una teoría con el sustento debido para la conformación de una ruta sistémica que permita el abordaje en próximos escenarios educativos, la generación de aspectos educativos y pedagógicos con fundamentos en las TIC y en los entornos tecnológicos con uso educativo. 


\section{REFERENCIAS CONSULTADAS}

1. Britio A., M., Jara Salazar, C., \& Larenas V. , C. (2009). El software educativo en el aula. Santiago de Chile. Recuperado a partir de http://bibliotecadigital.academia.cl/bitstream/handle/123456789/1945/tpeb719.pdf ?sequence $=1 \&$ isAllowed $=\mathrm{y}$

2. Fernández Cruz, F., Fernández Díaz, M., \& Rodríguez Mantilla, J. (2018). El proceso de integración y uso pedagógico de las TIC en los centros educativos madrileños. Educación XX1, 21(2). doi:https://doi.org/10.5944/educxx1.17907

3. Gómez, J. (2017). Experiencia en el diseño de instrumento para la virtualidad. Revista Arbitrada Interdisciplinaria Koinonía, 2(3), 65-79. Recuperado de https://fundacionkoinonia.com.ve/ojs/index.php/revistakoinonia/article/view/52/39

4. Hernández, R., Fernández, C., Baptista, P. (2014). Metodología de la Investigación. México: Editorial Mc - Graw - Hill Interamericana.

5. Parra Z., E., \& Pincheira J., R. (2019). Integración curricular de las TIC. Educación y Gestión del Conocimiento, 1-16. recuperado a partir de https://www.oas.org > cotep > GetAttach

6. Zevallos A., C. J. (2018). Competencia digital en docentes de una Organización Educativa Privada de Lima Metropolitana. LIma: PUCP. Recuperado a partir de http://tesis.pucp.edu.pe/repositorio/handle/20.500.12404/12942

7. Zurita, I. (2018). Gerencia estratégica como herramienta de transformación en la gestión educativa. EPISTEME KOINONIA, 1(2), 35-60. Recuperado de https://fundacionkoinonia.com.ve/ojs/index.php/epistemekoinonia/article/view/510

\section{REFERENCES CONSULTED}

1. Britio A., M., Jara Salazar, C., \& Larenas V., C. (2009). The educational software in the classroom. Santiago of Chile. Retrieved from http://bibliotecadigital.academia.cl/bitstream/handle/123456789/1945/tpeb719.pdf ?sequence $=1$ \&isAllowed $=\mathrm{y}$

2. Fernández Cruz, F., Fernández Díaz, M., \& Rodríguez Mantilla, J. (2018). The process of integration and pedagogical use of ICT in Madrid educational centers. Education XX1, 21 (2). doi: https: //doi.org/10.5944/educxx1.17907 
3. Gómez, J. (2017). Experience in instrument design for virtuality. Interdisciplinary Arbitrated Review Koinonía, 2 (3), 65-79. Recovered from https://fundacionkoinonia.com.ve/ojs/index.php/revistakoinonia/article/view/52/39

4. Hernández, R., Fernández, C., Baptista, P. (2014). Investigation methodology. Mexico: Editorial Mc - Graw - Interamerican Hill.

5. Parra Z., E., \& Pincheira J., R. (2019). Curriculum integration of ICT. Education and Knowledge Management, 1-16. recovered from https://www.oas.org scoteps GetAttach

6. Zevallos A., C. J. (2018). Digital competence in teachers of a Private Educational Organization of Metropolitan Lima. LIma: PUCP. Recovered from http://tesis.pucp.edu.pe/repositorio/handle/20.500.12404/12942

7. Zurita, I. (2018). Strategic management as a tool for transformation in educational management. EPISTEME KOINONIA, 1 (2), 35-60. Recovered from https://fundacionkoinonia.com.ve/ojs/index.php/epistemekoinonia/article/view/510 